Gefässchirurgie $2015 \cdot 20: 482-483$

DOI 10.1007/s00772-015-0085-7

c) Springer-Verlag Berlin Heidelberg 2015

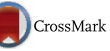

\author{
I. Flessenkämper ${ }^{1}$ A. Mumme ${ }^{2}$ \\ ${ }^{1}$ Klinik für Gefäßmedizin, HELIOS Klinikum Emil von Behring, Berlin, Deutschland \\ ${ }^{2}$ Katholisches Klinikum, Klinik für Gefäßchirurgie, Kliniken der Ruhr-Universität \\ Bochum im St. Maria-Hilf Krankenhaus, Bochum-Gerthe, Deutschland
}

\title{
Neue Therapiekonzepte bei der Behandlung des postthrombotischen Syndroms?
}

thrombotische Zustände nach partieller Rekanalisation bzw. persistierendem Verschluss. Folgerichtig verschlossen sie die insuffiziente $V$. femoralis distal des profundalen Einstroms. Oftmals wurden insuffiziente Stammvenen parallel gestrippt. Unter dieser radikalen Vorgehensweise wurden Ulkusheilungsraten von $80 \%$ beobachtet. Die in Europa parallel von Bauer um ca. 1948 entwickelte Therapie fand angeblich weltweit Anwendung [3]. Das theoretische Konzept der Umleitung des venösen Abstroms über klappentragende Stromgebiete erscheint auch heute noch durchaus nachvollziehbar [4]. Unvergessen sind auch die de-Weese-Clips, mit denen versucht wurde, den Reflux zu drosseln oder Lungenarterienembolien zu vermeiden. Sie resultierten regelmäßig in kompletten Rethrombosen [4].

Die Häufigkeit der Krankheit wurde von Schoop 1973 in Deutschland auf 1\% der erwachsenen Bevölkerung geschätzt [5]. In der Bonner Venenstudie lag die Häufigkeit des postthrombotischen Syndroms bei 3072 Probanden mit 1,1 \% exakt im selben Bereich. Bei Männern waren es $0,9 \%$, bei Frauen $1,2 \%$ [6].

Anhand der Daten des TULIPA-PLUSRegisters trat bei 24,5\% der Patienten mit tiefer Beinvenenthrombose (TVT) innerhalb von drei Jahren ein PTS auf, aber nur bei $1,5 \%$ ein schweres PTS. In diesem kurzen Zeitraum entwickelte kein Patient ein venöses Ulkus.

Wie hoch der Leidensdruck dieser Menschen und die soziale Bedeutung der Erkrankung ist, sieht man daran, dass die Betroffenen durchschnittlich 8 Jahre früher berentet werden und durchschnittlich 2 Monate im Jahr arbeitsunfähig sind [7].
Die zuvor genannten therapeutischen Ansätze wurden in den 1980er Jahren verfeinert. Es entwickelte sich eine rekonstruktive Chirurgie des PTS mit Ansätzen der Klappenrekonstruktion und -transposition bzw. -transplantation. Diese Entwicklungen wurden ausgebremst, als die niedermolekularen Heparine auf den Markt drängten und die ambulante Therapie der Thrombosen entwickelt wurde. Diese segensreiche Entwicklung, die tausenden von Patienten eine unnötige langwierige konservative stationäre Therapie ersparte, hatte den Nachteil, dass die Heparin-Studien als Endpunkt ausschließlich die Vermeidung der Lungenembolie im Fokus hatten, die Entwicklung des PTS aber weitgehend vernachlässigten.

\section{Was ist heute machbar?}

In den vergangenen 15 Jahren haben sich für Patienten mit PTS neue therapeutische Möglichkeiten ergeben. Neben der auch weiterhin für die meisten Patienten im Vordergrund stehenden konservativen Therapie existieren heute für beide Extremvarianten des PTS - die okklusive Form und die Refluxvariante - neue invasive Therapieoptionen. In den USA waren es Neglen u. Raju [8], die sich intensiv mit der endovaskulären Restitution chronisch postthrombotisch verschlossener Venen beschäftigten und fundierte wissenschaftliche Arbeiten zu diesem Thema veröffentlichten. Darüber hinaus leistete vor allem die italienische Arbeitsgruppe um Maletti u. Lugli [9] einen wesentlichen Beitrag mit der Entwicklung einer Operationstechnik zur Schaffung von Neoklappen aus der postthrombotisch geschädig- 
ten Gefäßwand. Mittlerweile sind es auch einige Kliniken in Deutschland, die sich intensiv mit Erkrankungen des tiefen Venensystems befassen. Dennoch gehören Patienten mit einem ausgeprägten PTS zu den am schlechtesten versorgten Patienten überhaupt. Dies auch im Hinblick auf die konservative Therapie, bei der es oftmals schon an einer suffizienten Kompressionsbehandlung mangelt. Rezepte werden aus pekuniärem Interesse abgeändert oder aufwendige Pelottenversorgungen den Patienten ausgeredet, bzw. nicht umgesetzt.

\section{》) Patienten mit ausgeprägtem \\ PTS gehören zu den am schlechtesten versorgten Patienten überhaupt}

Die Beschäftigung mit der Pathophysiologie des PTS, den konservativen und invasiven Therapieoptionen als Themenschwerpunkt in der aktuellen Ausgabe der Gefässchirurgie soll auf die Problematik der Behandlung des bislang wenig beachteten Krankheitsbildes aufmerksam machen. Auf der anderen Seite soll der Stand der rekonstruktiven Therapie am tiefen Venensystem dargestellt werden.

Ein Artikel von $V$. Wienert verdeutlicht uns die aktuellen Erkenntnisse über die Pathophysiologie des PTS. H. Jalaie et al. berichten über ihre Erfahrungen mit der Weiterentwicklung der aus den USA zu uns gekommenen interventionellen Ansätze. Erweiterte Darstellungsmöglichkeiten, neue Materialien mit verschiedensten Drähten, lenkbaren Schleusen und Katheter sowie der Entwicklung venöser Stents eröffnen neue Optionen in der rekanalisierenden Therapie des PTS. Darüber hinaus darf nicht vergessen werden, dass auch offen-operative Therapieoptionen bestehen, die möglicherweise zu selten angewandt werden. Kleinspehn et al. geben einen Überblick über die vorhandenen Optionen für die rekonstruktive Chirurgie an den Beinvenen.

Den Patienten mit ausgeschöpfter konservativer Therapie können die rekanalisierend-rekonstruktiven Methoden offenkundig eine Perspektive zur Linderung ihrer Beschwerden bieten. Es gilt daher, den bestehenden therapeutischen Nihilismus zu überwinden und die Behandlungsmethoden dieses häufigen Krankheitsbildes zu erweitern.

Wir stehen allerdings noch ganz am Anfang.

Ihre

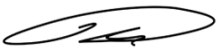

Dr. I. Flessenkämper

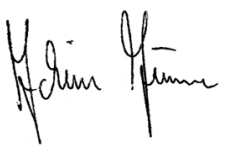

Prof. Dr. A. Mumme

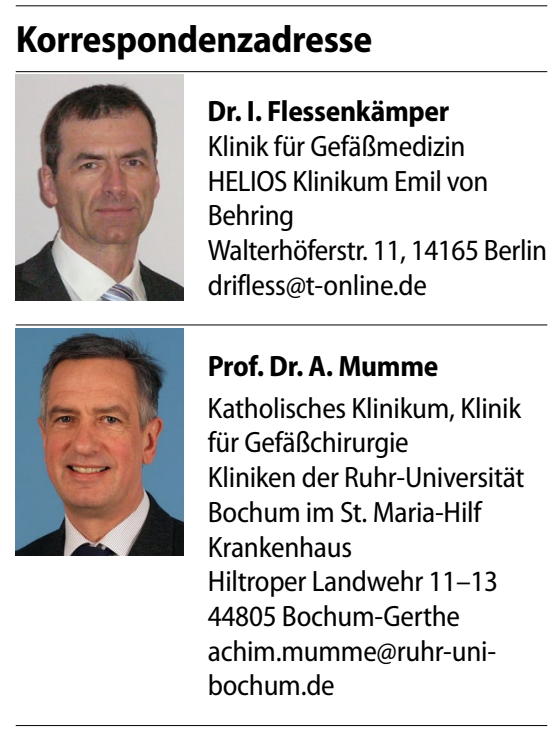

Interessenkonflikt. I. Flessenkämper und A. Mumme geben an, dass kein Interessenkonflikt besteht.

\section{Literatur}

1. Halse TH, Bätzner K (1951) Das postthrombotische Kreislaufsyndrom. Ätiologie, Diagnostik und Therapie. Med Welt 40:1243-1248

2. Halse TH (1954) Das postthrombotische Syndrom. Steinkopff, Darmstadt

3. Bauer $G$ (1948) The etiology of leg ulcers and their treatment by resection of the popliteal vein. J Intern Chir 8:937-967

4. Hach W (2006) Venenchirurgie. Schattauer, Stuttgart

5. Schoop W (1973) Postthrombotisches Syndrom. In: Hornbostel H, Kaufmann W, Siegenthaler W (Hrsg) Innere Medizin in Praxis und Klinik. Thieme, Suttgart

6. Rabe E, Pannier-Fischer F, Bromen K, Schuldt K, Stang A, Poncar CH, Wittenhorst M, Bock E, Weber $\mathrm{S}$, Jöckel K-H (2003) Bonner Venenstudie der Deutschen Gesellschaft für Phlebologie. Phlebologie 32:1-14
7. Wienert V (1999) Epidemiology of leg ulcers. Curr Probl Dermatol 27:65-69

8. Neglen P, Hollis KC, Olivier J, Raju S (2007) Stenting of the venous outflow in chronic venous disease: long-term stent-related outcome, clinical, and hemodynamic result. J Vasc Surg 46:979-990

9. Lugli M, Guerzoni S, Garofalo M, Smedile G, Maleti $O$ (2009) Neovalve construction in deep venous incompetence. J Vasc Surg 49:979-990 